\title{
Querría que me lo compruebes / comprobaras / comprobases: elección del tiempo verbal tras expresiones de volición atenuada en el español europeo ${ }^{1}$
}

\section{Querría que me lo compruebes / comprobaras / comprobases: verb tense choice after expressions of attenuated volition in European Spanish}

\author{
ANNA LÓPEZ SAMANIEGO ${ }^{1}$, ILPO KEMPAS ${ }^{2}$ \\ ${ }^{1}$ Filología Hispánica. Universitat de Barcelona. Barcelona, España. \\ Correo electrónico: alopezsam@ub.edu
}

${ }^{2}$ Filología Española. Universidad de Helsinki y Seinäjoki University of Applied Sciences, Finlandia. Correo electrónico: ilpo.kempas@seamk.fi

En este artículo, basado en un material empírico de 552 casos, se examina la realización temporal del verbo de la oración subordinada tras expresiones de volición atenuada con querer (quisieralquerríal quería) en el español peninsular, así como la variación en cuanto a la selección de la forma del verbo modal en estas estructuras. La hipótesis de partida es que el uso no canónico del presente de subjuntivo en estas construcciones se está extendiendo, especialmente, tras la forma quisiera. Se estudia la frecuencia real de dicho uso en muestras de discurso procedentes de Internet que representan un uso "oralizado" de la lengua (Yus 2010). Además, el análisis se extiende a las otras dos expresiones, para ver si la sustitución ocurre por analogía también tras ellas.

Palabras clave: español peninsular, concordancia de tiempos, subordinación, verbo querer, expresiones de volición atenuada

The present article, based on empirical material of 552 cases, focuses on the temporal realization of the verb of the subordinate clause after expressions of attenuated volition with querer (quisieral querrialqueria) in Peninsular Spanish. It also deals with variation in the choice of the form of the modal verb in these structures. The initial hypothesis is that the non-canonical use of the

\footnotetext{
$1 \quad$ Este trabajo se inserta dentro de un proyecto de investigación financiado por el Ministerio de Economía y Competitividad de España, en el que colabora el Prof. Kempas: Desarrollo conceptual y análisis empírico de la variación sintáctica en español: los objetos verbales y las estructuras de pasiva (FFI2013-41250-P), del que es IP la Prof. María José Serrano (Universidad de La Laguna).
} 
present subjunctive in these constructions is becoming increasingly common especially after the form quisiera. The article studies the real frequency of this use in online samples of discourse, which represent the "oralized" use of language (Yus 2010). In addition, the analysis is extended to the other two expressions, to find out whether this substitution also happens after them, by analogy.

Key words: Peninsular Spanish, tense agreement, subordination, verb querer, expressions of attenuated volition

\section{INTRODUCCIÓN}

La existencia de grandes corpus informatizados para el análisis del español, así como la riqueza de datos empíricos sobre el uso del lenguaje que ofrecen los buscadores de Internet han facilitado en los últimos años el análisis descriptivo de las alternancias en el uso de las formas verbales en español (entre pretérito perfecto y pretérito indefinido, por ejemplo), así como de correlaciones temporales no canónicas, distintas de las que se registran en las gramáticas normativas. Aunque parece esperable que esta variación sea menor en el caso de las construcciones parcialmente fijadas - como las peticiones corteses con verbos de volición atenuada <Quisieralquerríalquería + verbo en imperfecto de subjuntivo>-, el análisis de datos reales pone de relieve la existencia de variación también en estas construcciones.

\subsection{Objetivo del estudio}

La motivación de este estudio surge a partir de una clase del curso de Sintaxis verbal española impartido en la Universidad de Helsinki en la primavera de 2014 por uno de los autores, cuando un estudiante nativo español (peninsular) planteó que, en su opinión, <quisiera que> podía también ir seguido del presente de subjuntivo, cante (p. ej. quisiera que lo hagas), además de por la forma del imperfecto cantara / cantase propia de la concordancia temporal canónica (quisiera que lo hicieras/hicieses). El estudiante añadió que esta última concordancia (con la subordinada en imperfecto de subjuntivo) le parecía más natural introducida por <quería que> y <querría ques (quería / querría que lo hicieras / hicieses). Estas intuiciones desencadenaron el interés por estudiar la cuestión empíricamente.

En el presente artículo, por tanto, se analizará la elección de la forma verbal en subordinadas de subjuntivo cuando la oración principal está compuesta por expresiones de volición atenuada con el verbo modal querer, en concreto, con las variantes quisiera, quería y querría. Estas tres formas desempeñan, en principio, una misma función en dicho contexto, aunque con ligeras diferencias de matices, de modo que, en sentido amplio, pueden considerarse como alomorfos del condicional del verbo querer para la expresión de la petición cortés. Nos interesa, sobre todo, analizar las alternancias que se producen en el uso 
en cuanto a la realización temporal de la forma que aparece en subjuntivo después de estas expresiones modales.

En segundo lugar, nos interesa también analizar si existe variación en el uso de los alomorfos en - ra y -se del imperfecto de subjuntivo que aparecen en la cláusula subordinada de estas construcciones. Si bien el primero se considera como el más frecuente (NGLE 2010: \$24.2b) y en proceso de sustituir a las formas en -se, cabe plantearse si aumenta la frecuencia relativa de la variante cantase en algún contexto sintáctico específico. Este es el caso señalado por Kempas (2011: 254-255) para el siguiente contexto:

Hoy (verbo en un tiempo del pasado) ... al trabajo a las siete. (verbo en un tiempo del pasado) .... en coche para evitar que la lluvia me

En este contexto, en las nueve zonas geográficas incluidas en el estudio citado ${ }^{2}$ se registra un aumento en la frecuencia relativa de cantase $(45,2 \%)$ en comparación con los otros cuatro casos examinados (promedio 23,04\%). Resulta pertinente, pues, observar cuál es la frecuencia de aparición del imperfecto de subjuntivo en -se en las construcciones con verbos de volición atenuada.

Por último, queremos verificar si, en el corpus, las mujeres y los varones coinciden en sus elecciones entre la forma en -ra y la forma en -se. En Kempas, donde se examina la bibliografía sobre la variación entre cantara y cantase en Espańa, no se llega a una conclusión definitiva sobre las diferencias entre hombres y mujeres. Aunque en su estudio realizado en Granada, Valeš (2006) no había incluido esta variable, los resultados de Kempas (2011: 256) ponen de manifiesto una diferencia estadísticamente significativa entre mujeres y varones en solo dos de la totalidad de doce casos incluidos en las encuestas, además de un caso donde la diferencia se acerca al umbral de significancia $(\mathrm{p}=0,058)$. En estos tres casos, las mujeres utilizan la forma en -ra en mayor medida que los varones.

Como punto de partida del presente artículo, se han reanalizado los resultados de análisis del corpus oral de Val.es.co (Briz et al. 2002) presentados en Kempas (2011: 258), a los cuales no era posible aplicar la prueba de chi-cuadrado. El empleo del test exacto de Fisher arroja como resultado que, en efecto, en dicho corpus existe una diferencia significativa entre mujeres y varones $(\mathrm{p}=0,003)$ : en Valencia, las mujeres favorecen la forma en -ra mucho más que los hombres. Por consiguiente, este resultado coincide con los resultados anteriormente mencionados de Kempas que indicaban que las mujeres resultan favorecer la forma cantara (en ciertos contextos). Por ello, será interesante comprobar también si los casos recogidos para el presente estudio (procedentes de CREA y de búsquedas en Google) muestran una diferencia o no en este sentido (apartado 3). Nuestra hipótesis inicial, basada en los hallazgos mencionados, es que las mujeres muestran una preferencia por cantara, pero no está claro que la diferencia llegue a resultar estadísticamente significativa.

2 Los lugares de realización del estudio fueron Galicia (Vigo y Lugo), León, Madrid, Zaragoza, Tolosa, Santander, Barcelona, Castellón, Granada. 
López Morales (2004: 155) señala que las mujeres suelen ser más conscientes de la valoración de que su comunidad hace de los fenómenos de lenguaje y apoyan fenómenos de mayor prestigio social; este patrón se ha reconocido en muchos estudios, como Labov (1966). En consecuencia, si en el presente estudio se confirma que la forma en -ra es la preferida por mujeres, indica con mucha probabilidad que esta es la forma considerada como más prestigiosa frente a $-s e$.

\subsection{Las variantes <quisiera que >, <queria que $>$ y <querria que>}

Según las gramáticas normativas, las variantes <quisiera que>, <quería que> $\mathrm{y}<$ querría que $>$ requieren que el predicado de la subordinada que les sigue aparezca en imperfecto de subjuntivo, cantaral cantase (p. ej., Butt y Benjamin 2004: 283-284). Ello se debe a que estas tres formas se relacionan con la llamada esfera del pasado, si bien el tiempo de realización del evento de querer es factualmente el presente. Sarmiento y Sánchez (1989: 146) atribuyen el uso del imperfecto de subjuntivo tras <querría que> a la asimilación del condicional al modelo de tiempos pasados. En efecto, como ejemplo de esta vinculación del condicional con las formas del pasado puede plantearse la analogía con el periodo hipotético, donde el condicional en apódosis coaparece con el imperfecto de subjuntivo en prótasis (p. ej. lo haría si pudiera / pudiese). Cabe notar, además, que el condicional contiene la terminación del imperfecto (-ía-). No obstante, justamente en el caso del condicional en construcciones como la que nos ocupa (seguido de un verbo referido a un tiempo posterior en la cláusula subordinada), la vinculación con las formas del pasado es menos evidente que cuando el verbo de volición aparece en los otros tiempos posibles (quería, quisiera).

En el marco de la teoría sobre la temporalidad verbal de Rojo (1978), la aparición de estas formas temporales pertenecientes a la esfera del pasado para describir un evento que se desarrolla en el presente constituye un uso dislocado de tales formas, que comporta una sustitución del valor temporal que les es propio por un valor modal que, en el caso de las formas de pasado, se caracteriza como un valor de irrealidad. Para el caso específico que nos ocupa, el de los verbos modales que expresan volición atenuada, este valor modal se interpreta concretamente como un valor de cortesía. En gran parte de sus empleos, las estructuras analizadas vehiculan actos de habla directivos en beneficio del oyente (Albelda $e t$ al. 2014: 50) que requieren el empleo de estrategias de atenuación o de cortesía mitigadora dirigidas a prevenir una posible amenaza a la imagen del destinatario (ibid.: 20). Aplicando la metodología para el análisis pragmático de la atenuación propuesta por Albelda et al. (2014), el verbo de volición, expresado mediante usos dislocados de las formas verbales que expresan baja "intensidad obligativa" (Albelda y Barros 2013: 40), actúa en estos casos como atenuante, en tanto que la forma verbal subordinada forma parte del contenido atenuado.

En lo que respecta a la frecuencia general de uso de las variantes <quisiera que $>$, <quería ques y <querría ques, podemos suponer a priori que <quisiera que $>$ y <querría 
que> son las más frecuentes, porque <quería que> es de uso más restringido, relacionado principalmente con solicitudes y sugerencias dirigidas a un receptor (NGLE 2010: \$23.11g). En cambio, <quisiera que> y <querría que> pueden aparecer además en otros contextos, como en simples expresiones de preferencia, desprovistas de un deseo de obtener lo deseado de forma inmediata (de tipo 'me gustaría si o 'ojalá que': Quisiera/Querría ser tan alta como la luna).

Por otra parte, el uso del imperfecto de subjuntivo con este valor de cortesía también está sometido a restricciones. A diferencia de la alternancia del imperfecto cantaba con el condicional, que se da en contextos de cortesía con verbos más diversos, como en ¿No podíamos/podríamos ir a otro sitio? o Deseaba/Desearía realizar una llamada (Rojo 1978: 119; Serrano 2006: 124; NGLE 2010: \$23.11e y ss.), la selección del imperfecto de subjuntivo en estos contextos se limita prácticamente a los verbos modales querer y deber $\left({ }^{*}\right.$ ¿No pudiéramos ir a otro sitio?, *Deseara realizar una llamada).

Sobre la frecuencia de uso de cada una de las variantes <quisiera que $>$ y <querría que>, cabe decir que ambas formas alternan en el español general (NGLE 2010: 23.15u). Dado que el empleo de la forma quisiera en estos contextos constituye un resto de las estructuras condicionales ya en desuso del tipo Si fuera conveniente lo dijera, con imperfecto de subjuntivo en la apódosis (Rojo 1978: 126), se podría especular que esta sería la forma de uso más restringido. De hecho, las gramáticas suelen tratarla, frente al imperfecto de indicativo y al condicional, como una variante marcada, "más propia de un lenguaje cortés y elaborado" (Rojo y Veiga 1999: 2917).

Aun así, quisiera parece ser una notable excepción, una forma que se ha mantenido con firmeza en este uso como una expresión fija, hasta superar en frecuencia a querría. Basándose en los materiales del "Proyecto de Habla Culta", relacionado con el habla de las principales ciudades del mundo hispánico, DeMello (1997: 43) registró para querría un total de 16 casos $(8,1 \%)$ y para quisiera $181(91,9 \%)$, respectivamente $(\mathrm{n}=197)$.

\subsection{Sobre la concordancia temporal}

El español europeo suele observar estrictamente la concordancia temporal, que en el caso que nos ocupa, significa el principio según el cual el tiempo de la subordinada de subjuntivo debe representar la misma esfera temporal que el predicado de la oración principal que le precede (p. ej., quiero que lo hagas, pero queríalquise que lo hicieras/hicieses). Como ya hemos visto en el apartado 1.1, la concordancia temporal abarca también los casos en los que el predicado de la oración principal esté en condicional -expresado, en el caso del verbo querer, por las variantes querría, quisiera y quería y la subordinada esté orientada hacia el futuro. Por consiguiente, según la gramática normativa y el uso establecido, el subjuntivo de la subordinada debe estar en una forma de pasado (cantaralcantase).

En efecto, este es el patrón prevalente en las lenguas románicas en general, por ejemplo: 
Catalán: Voldria que em diguessis...

Italiano: Vorrei che mi dicessi...

El francés es una excepción; como en este idioma el imperfecto de subjuntivo se ha sustituido predominantemente por el presente, esto concierne, asimismo, al condicional:

(2) Je voudrais que tu me dises...

No obstante, el uso del imperfecto de subjuntivo (je voudrais que tu me disses) sigue siendo gramatical, pero pertenece a un estilo literario y arcaico (Grevisse 1993: 1274).

También las variedades hispanoamericanas del español han experimentado una evolución semejante a la registrada en francés, esto es, la sustitución de cantara por cante. Según Aleza Izquierdo (2010: 153), ocurre en todos los niveles del habla. Los siguientes ejemplos, mencionados por Kany (1969: 221) ilustran la ruptura de la concordancia temporal en dos variedades hispanoamericanas:

(3a) Fui a verla para que me preste un libro. (En lugar de prestara / prestase). (Arg.)

(3b) Lo auscultaron uno tras otro, y mientras ellos discutían, el enfermo seguía hablando sin que ninguno le escuche ni lo tome en cuenta. (En lugar de escuchara / escuchase y tomara / tomase, respectivamente). (Ch.)

Por esta razón, en el presente análisis hemos dedicado mucho esfuerzo a asegurar que los casos estudiados provengan de hablantes o autores españoles.

En el apartado 1.1, se apuntaba la posibilidad de que, en el español europeo, la concordancia temporal haya sufrido una ruptura después de las expresiones de volición atenuada con querer, al menos con <quisiera ques. Se trataría de lo que Carrasco (1999: 3096-3097) denomina una concordancia de sentido: un caso en el que el verbo de la subordinada no tiene en cuenta el valor temporal original de la forma del verbo principal, sino el valor temporal secundario que este desarrolla en un contexto determinado. Aplicando este concepto a las estructuras de volición atenuada que aquí nos ocupan, se trata de casos en los que el verbo de la subordinada no tiene en cuenta el valor temporal primario de las tres formas que puede adoptar el verbo principal (todas pertenecientes a la esfera del pasado), sino el valor secundario que estas desarrollan al aparecer en el contexto de una petición cortés, esto es, un valor de presente; de ahí que pueda aparecer también, como aquí se pretende mostrar, un presente de subjuntivo en la subordinada.

Si la concordancia temporal empieza a romperse también en el español europeo (dando lugar a concordancias de sentido, no canónicas), es natural que esto ocurra primero 
en un contexto en el que, pese a emplearse una forma tradicionalmente asociada con la esfera del pasado, el predicado está orientado hacia un evento futuro. En realidad, el predicado de la principal en <quisieralqueríalquerría que lo hagas>, en su uso temporalmente dislocado en el que la frase se refiere al momento presente, está claramente a la formulación con presente $<$ quiero que lo hagas . Es posible que, si llega a constatarse como un uso establecido en la variedad que nos ocupa, el uso de cante tras <quisieralqueríalquerría que $>$ pueda explicarse por concordancia de sentido o incluso por analogía con <quiero que $+\mathrm{V}$ en presente de subjuntivo>.

\subsection{Las variantes cantara $y$ cantase}

Son muchos los investigadores que consideran que cantara y cantase son alomorfos en variación libre, especialmente en el español europeo (Rojo y Veiga 1999: 2910). Otra opinión prevalente, relacionada con la premisa anterior, es que, cuando cantara y cantase funcionan como alomorfos, no comportan ninguna variación contextual. Entre autores que comparten esta postura figuran, por ejemplo, Alarcos Llorach (1994: 158), Lorenzo (1980: 248), Urrutia Cárdenas (1988: 269) y Ridruejo (1999: 3216). Así, en muchas presentaciones de la gramática española, ambos alomorfos se estudian juntos con sistematicidad; como ejemplo cabe mencionar las numerosas obras de Veiga, como Rojo y Veiga (1999) o Veiga y Mosteiro Louzao (2006).

Una opinión contraria a la hipótesis predominante de que todo tipo de variación contextual está excluida entre cantara y cantase fue presentada por Pottier, hispanista francés (1969: 38). En su opinión, cantase predomina cuando el evento ha tenido lugar o está muy planificado, y cantara es más frecuente cuando su realización es más incierta. También Schmidely (1992: 1310) llega a la conclusión de que ambas formas presentan diferencias de significado, pero su interpretación no concuerda con la de Pottier, siendo más bien contraria a esta última. Según Schmidely (loc. cit.), cantara corresponde, la mayoría de los casos, a lo cierto de una hipótesis, al simple deseo, a las opiniones corrientes, a lo más objetivo, mientras que cantase se reserva para lo fuertemente hipotético, lo extraordinario, lo más chocante, lo inadmisible, lo distante, lo "difícil de tragar". Aunque las conclusiones de Schmidely se basan en el análisis de una única obra, Cinco horas con Mario de Miguel de Delibes (1966), están -al menos en cierta medida- en línea con las observaciones de Serrano (2006: 139), relativas a las oraciones condicionales. Basándose en sus resultados de Tenerife (Serrano 1994), la autora concluye que, en estas construcciones, los hablantes introducen cantara cuando la hipótesis es o quiere presentarse como más probable, y cantase cuando es o quiere presentarse como menos probable. Ya hemos mencionado (apartado 1.1) que en Kempas (2011: 254-255) se registra un aumento en la frecuencia relativa de cantase en un contexto sintáctico específico, representado por tres casos diferentes, cuyo denominador común es que el evento en subjuntivo es hodiernal, anterior al momento de enunciación y-lo que puede ser el factor decisivo en este caso- no se ha producido (2011: 260). 
Los hallazgos mencionados permiten plantear, pues, que, aunque cantara sigue siendo globalmente la forma más frecuente, pueden existir contextos que favorecen la aparición de cantase, la forma menos frecuente. En el estudio de Kempas, en los tres casos, sus frecuencias son del 28,9, 33,6 y 45,2 por ciento: inferiores a las de cantara, pero más elevadas que en los demás casos examinados.

Cuando cantara y cantase están en libre variación, predomina cantara, tanto en España como en Hispanoamérica (NGLE 2010: \$24.2b; Rojo 1996). Además, en esta última zona, cantara se usa de forma aún más predominante o exclusiva en la lengua oral (p. ej. Kany 1969: 222; DeMello 1993: 236; Quesada Pacheco 2002: 124; cf. también Veiga 1996: 100-104 y Rojo 2011: 214). En lo que respecta a España, DeMello (1993: 236) señala para la forma cantara las frecuencias del 84 (Madrid) y 87 (Sevilla) por ciento de los casos.

En España, hay zonas que prefieren cantara en mayor medida que otras. Con la mayor certidumbre, como tales pueden señalarse Canarias (NGLE 2010: $\$ 24.2 b$; Serrano 1994) y Andalucía (Mondéjar 1970; Carbonero Cano 1990; Kempas 2011). En efecto, la NGLE (loc. cit.) incluye también el noroeste peninsular en este grupo, pero lo consideramos un error, porque en gallego, al igual que en portugués, la forma cantara está reservada únicamente al pluscuamperfecto, mientras que cantase expresa todas las funciones del imperfecto de subjuntivo. Además, los resultados empíricos de Kempas (2011: 254-255) demuestran que el foco del uso de cantase en el ámbito peninsular es justamente Galicia. Como otras dos zonas donde la frecuencia de cantase se muestra superior a las demás zonas peninsulares, cabe mencionar, sobre la base del estudio anterior, Cantabria (Santander) y Tolosa (País Vasco).

Ya hemos visto (apartado 1.1) que faltan evidencias para postular unívocamente que existen diferencias entre hombres y mujeres en cuanto a la preferencia por una u otra forma. Sin embargo, en Valencia (cf. supra) las mujeres parecen favorecer la forma en -ra mucho más que los hombres. Los resultados del presente estudio pueden contribuir a arrojar luz sobre este interrogante.

Aparte de la posible variación entre hombres y mujeres en el uso de las dos variantes, también resulta polémica la idea de si cuestiones de prestigio pueden incidir en su frecuencia de uso por parte de diferentes grupos socioculturales. Es sabido que las diferencias socioculturales suelen manifestarse sobre todo en el área de la fonología. Aun así, López Morales (2004: 175) señala la existencia de la variación sociocultural también en el campo de la sintaxis; en lo relativo al español, menciona el estudio de Bentivoglio y D'Introno (1977) sobre el dequeísmo en Caracas. Serrano (1996: 137) concluye que (en Tenerife) cantase es más utilizado entre hablantes de mayor instrucción, y cantara, respectivamente, entre aquellos que pertenecen a grupos socioculturales más bajos. También Valeš (2006) llega a la conclusión de que la variante con más prestigio (en Granada) es justamente cantase. No obstante, Bejarano, cuyo artículo se publicó ya hace más de cincuenta años (1962), tiene una opinión opuesta: según él, cantara es usado por personas cultas, sobre todo universitarios, mientras que cantase pertenece a la lengua literaria y -lo que parece muy paradójico- a "las personas que no han estudiado, sobre todo si viven en los pueblos o en el 
campo". Sea cual sea la verdad, lo cierto es que la sociedad y las preferencias han cambiado mucho durante los decenios pasados.

\section{Método y materiales}

A fin de analizar el grado de variación en el uso de las estructuras con verbos de volición atenuada, utilizando el buscador Google, recogimos todas las apariciones de las frases exactas <quisiera que me>, <quería que me> y <querría que me> disponibles en las páginas de Internet españolas (site:es) en un mismo momento de observación, esto es, del 12 al 16 de diciembre de 2015. La mayor parte de los ejemplos localizados corresponden, como era de esperar, a géneros de tipo interactivo, como intervenciones en foros, blogs y comentarios en diversas páginas web. Además, las frases anteriores se combinaron, -en otra búsqueda realizada en las mismas fechas-, con las formas cantara y cantase del imperfecto de subjuntivo y la del presente de subjuntivo del verbo decir en la segunda persona de singular (p. ej., quisiera que me dijeras/dijeses/digas). Incluimos el pronombre me en la frase de búsqueda por dos razones. En primer lugar, su presencia asocia la situación con más firmeza con una petición personal, en la que se insiste con mayor fuerza en que se cumpla la acción deseada. En segundo lugar, la aparición del pronombre prolonga la frase y limita así el número total de casos, para permitir la obtención de una enumeración completa.

El objetivo de una enumeración completa, ligada a un periodo delimitado, era obtener una idea sobre las frecuencias globales de estas tres formas sinónimas y poder constatar en qué orden de frecuencia aparecen; es de suponer que el resultado obtenido es extrapolable al uso general de la lengua. Queríamos determinar también con qué frecuencia aparece el presente de subjuntivo tras <quisiera que> en comparación con el imperfecto, posibilidad que en el espańol europeo será reconocida por cualquier hablante nativo, pero que es contraria a la concordancia temporal canónica de estas estructuras. Asimismo, nos interesaba ver en qué medida ese uso se había extendido, por analogía, a las formas sinónimas de <quería que> y <querría que>.

Por otro lado, nos interesaban también las frecuencias de cantara y cantase, respectivamente, para ver si la elección de cierta forma en la oración principal favorece cierta forma en la subordinada de subjuntivo, o, en concreto, si una de las formas favorece el mayor uso de la variante -se, considerada como la menos frecuente.

El verbo decir figura como punto de comparación porque, además de que es un verbo muy común, en los casos en cuestión se le dirige una petición al interlocutor, a quien el hablante trata informalmente, en segunda persona.

$\mathrm{Al}$ recoger el corpus, se aplicaron los siguientes criterios para filtrar los resultados:

1) La acción deseada a la que se refiere la subordinada de subjuntivo es posterior al momento de habla; por ejemplo, están excluidos los casos donde quería expresa el pasado. 
2) Los casos son enunciados por un hablante o escritor en la primera persona de singular (yo quisiera que).

3) La construcción no forma parte del periodo hipotético. Al aparecer en el periodo hipotético (p. ej., si quisiera que te fueras, te lo diría), las formas en cuestión no se orientan hacia una acción futura con igual fuerza.

4) El verbo de la frase principal y el de la subordinada es de polaridad positiva. Los casos de polaridad negativa presentan grandes diferencias semánticas respecto a los de polaridad positiva y, por ello, merecen ser estudiados en otra ocasión.

5) El hablante es español, o, al menos, nada sugiere lo contrario. Se excluyeron los casos en que el escritor dice ser hispanoamericano o en que su uso de la lengua lo sugiere (vocabulario, alteraciones ortográficas que reflejan la pronunciación propia de esta variedad "hase cinco años que lo conosco" y cuestiones de contenido, como la mención de temas o realidades culturales pertenecientes al ámbito hispanoamericano, etc.).

6) El texto representa la lengua contemporánea. Entre los documentos producidos por las búsquedas, hay ocasionalmente textos más antiguos, como La Celestina (1499), que no han sido tenidos en cuenta.

En los documentos, solo registramos un caso por autor o hablante, aunque de este mismo hubiera varias ocurrencias. En cambio, las tablas 1 y 2 se basan en parte en los mismos casos, aunque no de forma predominante. Aunque lógicamente podría suponerse que las búsquedas basadas en <quisiera / quería / querría que me dijeras / dijeses / digas $>$ producirían necesariamente los resultados incluidos en las búsquedas <quisiera / quería / querría que me $+\mathrm{V}>$, este no fue el caso. En efecto, estas últimas comprenden solo una pequeña parte de los ejemplos localizados en las primeras. Como explicaciones pueden plantearse las modificaciones en la disponibilidad de los documentos en la red durante el lapso de tiempo entre la primera búsqueda y la segunda o el hecho de que diferentes combinaciones de palabras producen un distinto número de resultados y favorecen la aparición de ciertas páginas.

Para completar las búsquedas realizadas con Google, buscamos en el corpus CREA todos los casos de <quisiera / quería / querría que mes que cumplían los criterios anteriormente especificados. Obtuvimos un total de 22 casos, y completamos después el panorama con una prueba adicional de 12 casos (total=34). Después de esta prueba, realizamos, por último, una búsqueda más con Google, con 27 casos.

Los casos incluidos en el corpus de las tres variantes son 491 en total; cuando a esto se suman 34 casos provenientes del CREA y los 27 casos de una prueba adicional con Google ${ }^{3}$, se llega a un total de 552 casos. Registramos el sexo del hablante o autor

Realizada con la construcción <quería que junto con el adverbio ahora. 
en todos los casos en los que era posible $(n=386 ; 74,7 \%)^{4}$. Un total de 186 enunciados $(48,2 \%)$ son producidos por un hablante o escritor femenino y 200 , por uno masculino $(51,8 \%)$. Muchas veces, esto implicaba "trabajo de detective", una búsqueda de marcas del género, como el uso de adjetivos referidos al propio autor. Si en un foro un autor usaba un avatar femenino o masculino, suponíamos que correspondía a su sexo real. Por otra parte, si no encontrábamos ninguna prueba del sexo del hablante o autor, lo dejábamos abierto. Cabe notar que, en los diálogos de cuentos ficticios, poco numerosos en nuestro corpus proveniente de Google, se registró también el sexo del hablante ficticio: nuestra premisa era que, si las mujeres y los varones, de hecho, difieren en su uso de las tres variantes, esto se reflejaría también en las elecciones realizadas para ellos por los autores de los cuentos, aunque ocurra inconscientemente.

Los resultados se presentan en el apartado siguiente. Los textos de los ejemplos están sin editar ni corregir, para conservar la autenticidad de cada ejemplo.

\section{Resultados}

Los resultados de las primeras búsquedas en Google con < quisiera / quería / querría ques produjeron 292 casos. Cabe mencionar que, en la absoluta mayoría de ellos, se trata de una situación en la que el hablante desea directamente que el interlocutor o receptor (o su representante) realice una acción; se trata, pues, de peticiones (<quisiera que me>: 95,7\%, <quería que me>: 86,1\%, <querría que mes: 85,1\%). Los tiempos de los verbos que siguen a las construcciones anteriores se presentan en la siguiente tabla:

Tabla I: <Quisiera / quería / querría que> + V.

\begin{tabular}{|l|c|c|c|c|c|}
\hline & $\begin{array}{c}\text { Imperf. de subj. en -ra } \\
(\%)\end{array}$ & $\begin{array}{c}\text { Imperf. de subj. en -se } \\
(\%)\end{array}$ & $\begin{array}{c}\text { Presente de subjuntivo } \\
(\%)\end{array}$ & Total & $\%$ \\
\hline Quisiera que me & $85(73,9)$ & $6(5,2)$ & $24(20,9)$ & 115 & 39,4 \\
\hline Quería que me & $26(72,2)$ & $6(16,7)$ & $4(11,1)$ & 36 & 12,3 \\
\hline Querría que me & $96(68,1)$ & $41(29,1)$ & $4(2,8)$ & 141 & 48,3 \\
\hline & & & & 292 & 100 \\
\hline
\end{tabular}

\footnotetext{
$4 \quad$ Los casos del CREA no se analizaron desde la perspectiva del sexo del hablante, porque la mayoría de las veces no era posible de determinarlo con seguridad debido al tamańo reducido del cotexto en torno a los casos examinados. Además, al tratarse en muchos casos de textos literarios, se hace difícil decidir si tener en cuenta el sexo del personaje o el del escritor o escritora como posible factor determinante de la variedad empleada.
} 
En primer lugar, vemos que la variante <querría que me> resulta la más frecuente, con 141 ejemplos, lo que corresponde al 48,3 por ciento de la totalidad de los casos. Aparece seguida por <quisiera que me>, con 115 casos (39,4\%). Se puede concluir, por tanto, que estas dos variantes son las principales y que <quería que me> es de uso más restringido en este tipo de contextos.

Cabe notar que, por lo general, el imperfecto de subjuntivo resulta el tiempo más frecuente de la subordinada en las tres variantes, como era de esperar.

(4a) Hola cielo. Quisiera que me dijeras ${ }^{5}$ que amuleto debo de llevar para tener mas fluidez de comunicacion y tener mas confianza en mi misma. Mi fecha de nacimiento es el 02-09-1967. Un millon de gracias.

(4b) Quería que me gustase el Nexux 5X, de verdad, lo quería por encima de todas las cosas.

(4c) Para terminar esta entrevista, querría que me diera su punto de vista sobre la polémica que hemos estado viendo últimamente en los medios sobre la diferencia en financiación entre los puertos de Santa Cruz y Las Palmas, y la negativa, por parte de Puertos del Estado, a permitir una autofinanciación de Puertos de Las Palmas.

No obstante, vemos que en el caso de <quisiera que me>, la frecuencia del presente de subjuntivo (cante) sube al 20,9 por ciento, a saber, a aproximadamente la quinta parte de los casos.

(5a) Has estado un tiempo formándote antes en la escuela, y me gustaría saber más cosas de ese aprendizaje. Además, quisiera que me digas cómo puedo ayudarte en tus primeros días aquí.

(5b) Hola Cristina,felicidades por tu programa, quisiera que me ayudes diciendome el nombre y donde puedo conseguir el serun natural que comentaste el otro dia, soy de Madrid.

(5c) Buenos dias quisiera que me faciliten la clave de inscripcion del curos online de incontinencia urinaria.

Por otro lado, como se ve, cante aparece también después de <quería que me> y $<q u e r r i a q u e$ me>, si bien con frecuencias considerablemente inferiores:

\footnotetext{
5 Los ejemplos se reproducen fielmente, sin corregir los errores de normativa que se producen en los textos originales
} 
(6a) Hola Coco solo queria que me pongas un apodo me lamo nadia.

(6b) Queria que me confirmen sobre un pedido que hice.

(6c) Querría que me hable de la colaboración entre los dos partidos.

Sobre la base de este resultado, puede plantearse si esta concordancia temporal de los ejemplos de (5) con la forma cante debería considerarse aceptable desde la gramática normativa. Con las otras dos variantes $(6 a-6 c)$, se emplea mucho menos. Aun así, queda demostrado que su uso se ha introducido también en esos casos, posiblemente por analogía con lo ocurrido con <quisiera que . El uso del presente de subjuntivo en estos últimos casos explica en parte que, al aplicar la prueba de chi-cuadrado, se registre una diferencia estadísticamente significativa entre los resultados para las tres variantes $\left(\chi^{2}=39\right.$, $p=0,000 ; d f=2)$.

El segundo resultado de interés se relaciona con la forma <querría que me>. Como se ve, la forma cantase, en general menos frecuente que cantara, resulta en este caso sorprendentemente frecuente $(29,1 \%)$; con exclusión de los casos de cante, sube al 29,9 por ciento. Significa, pues, que aparece en casi la tercera parte de los casos.

(7a) En primer lugar, querría que me describiese usted su primer recuerdo.

(7b) He cambiado de tarjeta. Y querría que me devolviesen el dinero de un evento cancelado.

(7c) Querría que me recomendase los fármacos que puedan evitar que la artrosis vaya a más, o que puedan ralentizar su avance, yo ya hago deporte y cuido mi alimentación. Muchas gracias.

Entre mujeres $(\mathrm{n}=74)$ y varones $(\mathrm{n}=114)$ no hay diferencia significativa en la elección entre -ra y - se $\left(\chi^{2}=1,1, \mathrm{df}=2, \mathrm{p}=0,3\right)$. No obstante, los resultados muestran que las mujeres prefieren cantara en algo mayor medida que los hombres (82,7 frente al 76,3\%), aunque esta diferencia no es estadísticamente significativa, por lo que no parece posible generalizar este resultado a la totalidad de los hablantes españoles.

Tampoco existe diferencia significativa entre mujeres y hombres en la elección entre cantara / cantase y cante $\left(\chi^{2}=3, \mathrm{df}=2, \mathrm{p}=0,08\right)$. Como el número total de casos de cante en los que se sabe el sexo del escritor o hablante es tan solo de 24 , no resulta posible extraer tendencias subyacentes en la actuación de las mujeres y hombres. Cabe mencionar, sin embargo, que $14(58,3 \%)$ de las apariciones de cante provienen de mujeres y $10(41,7 \%)$, de varones.

La tabla II ilustra lo que ocurre al añadir la forma de la segunda persona de singular del verbo decir (dijeras, dijeses, digas) a la frase anterior. 
Tabla II: <Quisiera / quería / querría que me> + verbo decir en $2^{\text {a }}$ persona de singular.

\begin{tabular}{|l|c|c|c|c|c|}
\hline & Imperf. de subj. en -ra & Imperf. de subj. en -se & Presente de subjuntivo & Total & $\%$ \\
\hline Quisiera que me & $66(64,7)$ & $21(20,6)$ & $15(14,7)$ & 102 & 51,3 \\
\hline Quería que me & $32(50)$ & $30(46,9)$ & $1(3,1)$ & 63 & 31,7 \\
\hline Querría que me & $23(67,6)$ & $10(29,4)$ & $1(2,9)$ & 34 & 17,1 \\
\hline & & & & 199 & \\
\hline
\end{tabular}

En primer lugar, se observan algunos importantes cambios en comparación con la tabla I. El más llamativo es el incremento general de la frecuencia de la forma cantase: es como si las otras dos variantes se acercasen en esto a <querría que me>, que exhibía este rasgo ya en la tabla I.

(8a) Hola Karlito,viendo tus modificaciones del sillín quisiera que me dijeses las medidas del agujero que le hiciste y de que manera esta centrado de atrás y adelante.

(8b) Tambien queria que me dijeses alguna crema o algo asi para algunos granitos que tengo en la cara y en los mofletes al lado de la nariz tengo como cicatrices moraditas y queria que me dijeses aver con que lo puedo quitar.

(8c) Querria que me dijeses que puedo hacer o que papeles tengo que entregar definitivamente ya que cada semana me requieren unos diferentes.

Por ello, como es de esperar, <quisiera que me> presenta una diferencia significativa respecto a la tabla anterior $\left(\chi^{2}=12,1, \mathrm{p}=0,002\right.$, cuando $\left.\mathrm{df}=2\right)$. Por el contrario, la segunda variante, <quería que me>, no permite el uso de la prueba de chi-cuadrado por el reducido número de casos de cante en dos celdas $(<5)$. Por esta razón, realizamos la prueba excluyendo los casos de cante, mirando solo la diferencia entre cantara y cantase en ambas tablas. Obtuvimos como resultado que $\chi^{2}=7,8$ y p=0,005 $(\mathrm{df}=1)$ : otra diferencia estadísticamente significativa. Hicimos otro tanto a la frase <querría que me>, por la misma razón. Como ya era de esperar, las distribuciones de esta frase no presentan diferencia significativa entre las dos tablas $\left(\chi^{2}=0,0, \mathrm{p}=1 ; \mathrm{df}=1\right)$.

En cuanto a los números totales de cada variante, se aprecia también un aumento en las frecuencias relativas de <quisiera que me> y <quería que me>, mientras que <querría que me> experimenta un retroceso. Puede plantearse si las dos variantes se perciben como más amables en este caso en que la petición se dirige a un representante de la segunda persona de singular. 
Aunque las distribuciones de la tabla II puedan parecer a primera vista más homogéneas entre sí que las de la tabla I, existe también en este caso una diferencia estadística significativa entre las tres variantes $\left(\chi^{2}=20,2, p=0,000 ; d f=2\right)$. Es explicable por la mayor frecuencia de cante tras <quisiera que me> y la de cantase tras <queria que me>. Examinamos también si la prueba muestra una diferencia estadísticamente significativa entre cantara y cantase con exclusión de los casos de cante. Otra vez, el resultado es significativo $\left(\chi^{2}=9,7\right.$, $\mathrm{p}=0,008 ; \mathrm{df}=1$ ). Queda confirmado, pues, que <quería que me> favorece con claridad la aparición de cantase, la forma normalmente menos frecuente, que alcanza la elevada frecuencia del 48,4 por ciento $(n=62)$.

(9a) Lo que quería que me dijeses es de qué otra forma pagas tú, que se me puede estar pasando mencionar o recordar...

(9b) Quería que me dijeses a que ciudades ha llegado el libro, porque a lo mejor donde he mirado yo, todavía no he llegado.

(9c) Hola Poul, quería que me dijeses que te parece el artista del aerógrafo Dru Blair y en concreto este retrato:

$\mathrm{Al}$ igual que para la tabla I, examinamos la posible diferencia entre hombres $(\mathrm{n}=52)$ y mujeres $(\mathrm{n}=83)$ en la elección de las formas cantara y cantase, esto es, <quería que me dijeras> frente a <quería que me dijeses $>$. Tampoco en este caso se registra una diferencia estadísticamente significativa entre $\operatorname{los} \operatorname{sexos}\left(\chi^{2}=1,7, \mathrm{p}=0,195 ; \mathrm{df}=1\right)$. No obstante, otra vez las mujeres resultan preferir la forma cantara en mayor medida que los hombres $(68,7$ frente al 57,7 por ciento), si bien, de nuevo, la diferencia no llega a ser significativa.

Por último, los casos de cante, <quería que me digas>, presentan un reparto muy igual entre hombres y mujeres. El sexo del hablante puede confirmarse en tan solo 13 casos, en los cuales cante aparece en mujeres seis veces y en hombres siete veces, respectivamente.

(10a) Quisiera que me digas, concretamente, si-como supongo- hay que presentar los originales a la censura previamente en doble copia. (enunciado por un hombre)

(10b) Estrella. Quería que me digas como te muestran las cartas que continuaremos con Sebas. (enunciado por una mujer)

(10c) Bueno solo querria que me digas como solucionarlo el tema de que tarda muchisimo poara ir a la calle, muchisimas gracias. (enunciado por un hombre)

A continuación, se presentan los resultados de la consulta del corpus CREA: 
Tabla III: Casos de <quisiera / quería / querría que me> en el CREA.

\begin{tabular}{|l|c|c|c|c|c|}
\hline & Imperf. de subj. en -ra & Imperf. de subj. en -se & Presente de subjuntivo & Total & $\%$ \\
\hline Quisiera que me & 10 & 4 & - & 14 & 63,6 \\
\hline Queria que me & 5 & - & - & 5 & 22,7 \\
\hline Querría que me & 1 & 2 & - & 3 & 21,4 \\
\hline & $16(72,7 \%)$ & $6(27,3 \%)$ & & 22 & \\
\hline
\end{tabular}

En primer lugar, se observa que el número total de casos $(n=22)$ es muy reducido en comparación con los obtenidos a través de búsquedas con Google $(n=491)$, resultado que confirma la utilidad de la red como fuente para obtener datos sobre el uso real de la lengua para los casos de construcciones, especialmente propias de contextos conversacionales y coloquiales, que aparecen con baja frecuencia incluso en los grandes corpus disponibles.

Llama la atención también la diferencia en el orden de aparición de las variantes: el CREA cuenta tan solo con tres casos de <querría que me>, lo que corresponde al 21,4 por ciento, mientras que esta variante era la más frecuente en la tabla I, con el 48,3 por ciento. Además, la forma cante está completamente ausente en la tabla III, incluso tras <quisiera que me>. Sobre la base de la tabla I, en la que su frecuencia es del 20,9 por ciento, se esperaría que en la tabla III hubiera 2-3 casos de esta variante.

El análisis de los casos pone de manifiesto una diferencia en el tipo del material: 15 de los 22 casos $(68,2 \%)$ provienen de textos ficticios (novelas y obras de teatro). En el corpus basado en búsquedas con Google, en cambio, solo hay tres textos de este tipo: la absoluta mayoría de los ejemplos están compuestos por consultas y peticiones espontáneas. Es natural que los diálogos de un texto literario, por auténticos que parezcan al lector, solo en raras ocasiones alcancen el grado de espontaneidad y naturalidad del discurso real.

Dado que en el CREA no hay ningún caso de cantase después de <quería que me>, decidimos extender el análisis a los casos <yo quería que>, con pronombre de primera persona explícito. Obtuvimos 12 ejemplos, en los cuales cantara aparece ocho veces $(66,7 \%)$ y cantase, cuatro veces $(33,3 \%)$. Aunque esta muestra es muy pequeńa, la frecuencia bastante elevada de cantase sugiere otra vez que entre <quería ques y la forma cantase puede haber una vinculación.

Sobre la base del análisis de los resultados del CREA, decidimos examinar la realización del predicado de la subordinada de subjuntivo tras <quería que> y realizamos una nueva búsqueda con Google (5 de enero de 2016), añadiendo el adverbio ahora a la frase para ayudarnos a encontrar más fácilmente casos orientados hacia el futuro. Recogimos todas las apariciones de esta frase en la primera persona de singular donde ahora quería que ocurre en el presente del hablante. Los resultados $(\mathrm{n}=27)$ se ilustran en la siguiente tabla: 
Tabla IV: Casos de <ahora quería que> y < Quería ahora que>.

\begin{tabular}{|l|c|c|c|c|}
\hline & Imperf. de subj. en -ra & Imperf. de subj. en -se & Presente de subjuntivo & Total \\
\hline $\begin{array}{l}\text { Ahora queria que / } \\
\text { Quería ahora } \\
\text { que }\end{array}$ & $15(55,6 \%)$ & $9(33,3 \%)$ & $3(11,1 \%)$ & 27 \\
\hline
\end{tabular}

Como se desprende de la tabla, cantara resulta la variante más frecuente, con el 55,6 por ciento de los casos. Es seguido de cantase, que presenta otra vez una frecuencia bastante elevada, apareciendo en la tercera parte de los casos $(33,3 \%)$. Si se excluyen los casos de cante, las frecuencias de cantara y cantase son del 62,5 y 37,5 por ciento, respectivamente.

(11a) Pero bueno ahora quería que pensases tu que pienses las horas de mi vida que me he pasado en tu servidor lo que me he divertido con vosotros desde que os conoci hasta hoy.

(11b) Sí que importa, ahora quería que cambiásemos los papeles...

(11c) Soy consumidor habitual de la gasolinera del Leclerc y del supermercado en el que me beneficio además de descuentos de $5 €$ por cada 40 de gasolina, pero ahora quería que me publicaran esta carta sobre las gasolineras.

Como acabamos de ver, también cante está representado, y, curiosamente, con un porcentaje idéntico a la de la tabla I $(11,1 \%)$.

(12a) Quería que la zona se pueda seguir utilizando de forma lúdica.

(12b) Lo pregunto porque esta opcion la he llevado a cabo y desde el panel de mi dominio he redirecionado a mi blog y ahora queria que este dominio sea el que aparezca en lugar del de bloger.

Por último, como los casos de la tabla IV son pocos, solo el test exacto de Fisher puede usarse para estudiar si entre hombres y mujeres hay una diferencia significativa en sus elecciones entre cantara y cantase. El resultado del test es que $\mathrm{p}=0,193$, de modo que no existe diferencia significativa entre los sexos. Aun así, al igual que en las tablas 1 y 2 , las mujeres prefieren la forma cantara. Por otro lado, los tres casos de cante provienen de varones. 


\section{ConClusiones Y Discusión}

El estudio empírico realizado ha permitido dar respuesta a los principales problemas presentados en el apartado 1.1. El primero de ellos era la posibilidad de uso de cante, además de cantare / cantase, tras <quisiera que> y, por ende, la ruptura de la concordancia temporal canónica, que supone que a una forma verbal de la esfera del pasado en la principal (cantaría, cantara, cantaba) le siga en la subordinada de subjuntivo una forma perteneciente a la misma esfera (cantara, cantase). Queda demostrado que, en efecto, este cambio se está produciendo en la variedad ibérica del español, aunque cabe plantearse si es lo suficientemente común como para considerarse una variante normativa. La mayor frecuencia aquí medida para la forma cante es del 20,9 por ciento, esto es, del orden de la quinta parte de los casos (tabla I), y que el segundo resultado es del 14,7 por ciento (tabla II), mientras que no aparece ninguna vez en la tabla III, compuesta manifiestamente por casos provenientes de diálogos literarios $(68,2 \%)$. Su ausencia en esta última tabla es comprensible, además de por el número reducido de las concordancias obtenidas, porque, aunque un escritor tienda a escribir en una lengua lo más actual y coloquial posible, es poco probable que contenga usos de gramaticalidad cuestionable. Estos deben aparecer primero de forma recurrente en el discurso espontáneo. De todas formas, este es, probablemente, un cambio en curso, que bien podría llegar a tener repercusiones en la gramaticografía en el futuro.

Por otro lado, aunque el mismo cambio se observa también después de <quería que> y <querría que>, en estos últimos casos la frecuencia de cante disminuye con claridad. Esto es llamativo sobre todo en la tabla I, donde, pese a la frecuencia algo superior de <querría que> a la de <quisiera que>, la de cante es de tan solo 2,8 por ciento (frente al 20,9 por ciento con la forma $<q u i s i e r a>$ ). Algunas causas concurrentes que podrían explicar esta mayor facilidad de la estructura de volición encabezada por <quisiera que> para favorecer la ruptura de la correlación verbal canónica son, de un lado, el carácter formulaico o fosilizado de esta forma verbal, que no admite sustitución por otras de significado similar (como * deseara que) y, de otro, el carácter excepcional de la construcción resultante: una cláusula independiente con el verbo principal en subjuntivo ${ }^{6}$.

La segunda cuestión mencionada en el apartado 1.1 se relaciona con la posible variación debida a factores contextuales en la elección entre cantara y cantase. Hemos visto (apartado 3) que la adición del pronombre personal me a <quisiera que > y <quería que> y la limitación de los casos a los de segunda persona de singular del verbo decir hace aumentar la frecuencia de cantase de forma estadísticamente significativa (tabla II). No obstante, este aumento no se produce en el caso de <querría ques, que ya presenta una mayor frecuencia de combinatoria con cantase en la tabla I, en comparación con las otras dos variantes. Por consiguiente, queda confirmado que sí existe variación contextual, resultado que desafía la hipótesis contraria predominante (apartado 1.4). No obstante, en ninguno de los casos llega 6 Agradecemos estas sugerencias de explicación del fenómeno a uno de los revisores anónimos de este
trabajo. 
a invertir el orden de frecuencia de aparición de cantara y cantase, donde predomina cantara. Este resultado se corresponde con lo señalado en Kempas (2011), esto es, que la variación contextual existe en determinados contextos en el seno de la variación libre, y se manifiesta de tal manera que cierto contexto puede favorecer una u otra forma, no obstante, sin que cantase llegue a ser predominante. En efecto, esta interpretación podría verse como una especie de compromiso entre la escuela que opina que los factores contextuales no influyen nada en la aparición de una u otra forma y la escuela que opina que los dos alomorfos expresan valores bien distintos.

Como explicación al considerable aumento de la frecuencia de cantase en la tabla II, se nos ocurre la posibilidad de que esta forma se escoja porque se percibe como más cortés que cantara. De hecho, tal hipótesis es consistente con el hecho de que se trate de la forma marcada, relacionada con lo más distante o más hipotético (cfr. 1.4). En todos los ejemplos recogidos en la tabla II está presente un interlocutor, -si bien virtualmente, en este caso-, y se trata de una petición con el objetivo de obtener alguna información específica. Como es bien sabido, las expresiones de volición difieren en cuanto a su intensidad y concreción, y en los casos de la tabla II, el nivel de ambas características es muy alto. En las situaciones de esta tabla, es, pues, natural que el hablante invierta un especial esfuerzo en atenuar el grado de impositividad del mensaje.

La relación que se está defendiendo aquí entre cantase y la expresión de un elevado grado de cortesía podría explicar, asimismo, el frecuente uso de cantase tras <querría que> (tabla I). Planteamos, por tanto, la hipótesis de que, a diferencia de quisiera y quería, que actúan como marcas de cortesía específicas para estas construcciones (cfr. 1.2), en el caso de <querría que + verbo> el hablante puede intuitivamente tratar de suavizar más su petición escogiendo cantase, la variante que se percibe como más distanciada del uso habitual y espontáneo de la lengua.

El tercer interrogante mencionado en el apartado 1.1 son las posibles diferencias entre hombres y mujeres en la elección entre cantara y cantase, y, con tal efecto, realizamos un reanálisis del corpus Val.es.co (Briz et al. 2002) con el test exacto de Fisher, presentado originariamente en Kempas (2011: 258). Este análisis mostró una diferencia significativa entre hombres y mujeres, en el sentido de que las mujeres favorecen la forma cantara mucho más que los hombres. En la misma ocasión, mencionamos los demás resultados de Kempas (2011: 256), basados en materiales peninsulares distintos de los del corpus Val.es.co, que ponen de manifiesto una diferencia significativa entre hombres y mujeres en solo dos de doce casos, pero que muestran la preferencia más acusada por cantara en mujeres.

No obstante, al aplicar las pruebas de chi-cuadrado sobre los datos de las tablas I y II (apartado 3) no se han observado diferencias estadísticamente significativas entre ambos sexos, como tampoco ha ocurrido al aplicar el test exacto de Fisher sobre la tabla III. No obstante, el resultado que se repite de forma consistente en todo lo anteriormente expuesto es la preferencia de la forma cantara entre mujeres. Por ello, a pesar de que no es posible mostrar una diferencia estadística en todos los casos, la base del conjunto de las pruebas analizadas refuerza la tesis de que las mujeres prefieren cantara en mayor medida que los 
hombres. En efecto, cabe recordar lo que significa el valor p. Este ayuda a determinar si una hipótesis es correcta o no. Si el valor p de un conjunto de datos está por debajo de cierto nivel (en este caso 0,05$)$, se rechaza la "hipótesis nula" $\left(\mathrm{H}_{0}\right)$. En otras palabras, se descarta la hipótesis de que los mismos resultados podrían obtenerse también por azar. En los resultados analizados la diferencia entre hombres y mujeres no es tan marcada como para que una prueba estadística permita, en todos los casos, excluir el azar $\left(\mathrm{H}_{0}\right)$ como explicación alternativa. Esto no significa, no obstante, que dicha diferencia no exista; por el contrario, si los resultados sobre varios conjuntos de datos producen sistemáticamente el mismo resultado, podemos concluir que, en un nivel general, esta diferencia sí existe. En consecuencia, en el español europeo, las mujeres tienden a usar cantara en mayor medida que los hombres, si bien este resultado no es generalizable a todos los hablantes.

En estudios sociolingüísticos, se ha confirmado que las mujeres, independientemente de su clase social, usan formas prestigiosas en estilos formales más a menudo que los hombres (Labov 1966; López Morales 2004: 155). Sobre la base de la mayor preferencia por cantara en mujeres, se podría suponer que esta es la forma más prestigiosa en el español europeo (recuérdese que es, además, la que la mayor parte de las gramáticas presentan como mayoritaria tanto España como en América). No obstante, cabe recordar la conclusión de Valeš (2006) (apartado 1.4) de que, en Granada, la forma de prestigio es justamente cantase.

El método de recogida de datos usado en el presente estudio, las búsquedas con Google, es todavía un enfoque bastante innovador, aunque cada vez más común ${ }^{7}$. En cuanto a la cantidad de ocurrencias que permiten extraer, las búsquedas con Google son claramente superiores a los corpus tradicionales, según hemos visto en el caso de la tabla IV. Como ejemplo del campo de los estudios sobre la lengua española, cabe mencionar los resultados mencionados por Sinner (2008: 248), donde el CREA produjo 196 ejemplos de la forma anduve, y una búsqueda con Google (google.es) 331 000, respectivamente. En Kempas (2015), se discuten las ventajas y desventajas de las búsquedas con Google en estudios lingüísticos y se detallan buenas prácticas a seguir para garantizar su validez y su fiabilidad.

En este sentido, es fundamental que las búsquedas con Google solo sirvan de base para la creación de un corpus y que el investigador no cometa el error de fiarse en frecuencias indicadas por el robot de búsqueda. Es necesario eliminar, entre otros resultados, las apariciones múltiples de ocurrencias y los casos en los que el fenómeno estudiado aparezca en el metatexto (como en foros de discusión sobre la corrección gramatical del fenómeno en cuestión), etc.

Una evidente desventaja de la obtención de datos por Internet es que los antecedentes de los hablantes o escritores suelen ser inaccesibles. Ya hemos mencionado en el apartado 2 que definir su sexo, por ejemplo, resultó una tarea laboriosa en este estudio; de hecho, en muchos casos, ha sido imposible. Por este motivo, las búsquedas con Google pueden

\footnotetext{
Por ejemplo, los autores de este artículo han publicado diversos artículos basados en parte en este enfoque (Kempas \& López Samaniego 2011, López Samaniego 2015) y uno basado únicamente en búsquedas con Google (Kempas 2013).
} 
ser poco apropiadas, por ejemplo, para determinados estudios sociolingüísticos, donde es importante tener acceso a todos los antecedentes de los hablantes (sexo, edad, educación, nivel de ingresos...).

A cambio, los buscadores de Internet ofrecen información de frecuencias de distintos usos en una población grande para analizar, por ejemplo, en qué medida se usa la variante $\mathrm{X}$ frente a la variante Y. Además, cada vez son más variados los usos de la lengua que pueden rastrearse mediante esta plataforma, que constituye el soporte de múltiples nuevos géneros y modos de comunicación. Dado que las pantallas constituyen ya un "tercer medio" (Crystal 2001), a caballo entre la oralidad y la escritura, pero a la vez distinto de estas dos, la inclusión de ejemplos procedentes de Internet en los estudios sobre comunicación debe ser necesariamente atendida por la lingüística del siglo XXI. Resulta, además, de especial importancia en los estudios sobre construcciones propias de la oralidad, ya que los expertos consideran la comunicación mediada digitalmente como un discurso "escrito oralizado" (Yus 2010).

Para terminar, el método "mixto" de obtención de datos del uso real de la lengua empleado en este estudio ha permitido comprobar la existencia de algunas tendencias de concordancia temporal que las gramáticas al uso no incluyen en sus descripciones. Como proyección futura de este estudio podría analizarse la realización del predicado de la subordinada de subjuntivo en parecidos casos en alguna(s) variedad(es) hispanoamericana(s), cuyos resultados podrían contrastarse con los del presente estudio. Es de suponer que, en Hispanoamérica, cante se usa con mayor frecuencia en los casos en los que en España (¿todavía?) prevalecen cantara y cantase. Un estudio contrastivo de este tipo permitiría plantear si la sustitución de cantaral cantase por cante representa un incipiente proceso de gramaticalización en el espańol europeo y si existen casos semejantes en otros contextos gramaticales.

\section{Obras citadas}

Alarcos Llorach, Emilio. 1994. Gramática de la lengua española. Real Academia Española. Madrid: España Calpe.

Albelda, Marta \& Barros García, Ma Jesús. 2013. La cortesía en la comunicación. Madrid: Arco Libros.

Albelda, Marta; Briz, Antonio; Cestero, Ana Ma; Kotwica, Dorota \& Villalba, Cristina. 2014. "Ficha metodológica para el análisis pragmático de la atenuación en corpus discursivos del español (es.por.atenuación)”. Oralia 17, 7-63.

Aleza Izquierdo, Milagros. 2010. “Observaciones gramaticales de interés en el español de América”. En Milagros Aleza Izquierdo y José María Enguita Utrilla (coords.): La lengua española en América: normas y usos actuales. Universitat de València, 95-223. Disponible en https://www.uv.es/aleza/esp.am.pdf [Consulta 1/1/2016].

Bejarano, J. V. 1962. "Sobre las dos formas del imperfecto de subjuntivo y el empleo de la forma en -se con valor de infinitivo". Filosofía y Letras 16, 77-86. 
Bentivoglio, Paola \& D’Introno, Franco. 1977. "Análisis sociolingüístico del dequeísmo en el habla de Caracas". Boletín de la Academia Puertorriqueña de la Lengua Española, 6, 1: 58-82.

Briz, Antonio y Grupo Val.Es.Co. 2002. Corpus de conversaciones coloquiales. Madrid: Arco/ Libros.

Butt, John \& Benjamin, Carmen. 2004. A New Reference Grammar of Modern Spanish. (Fourth ed.). McGraw-Hill.

Carbonero Cano, P. 1990. "Usos de las formas verbales en -ra y en -se en el habla de Sevilla (nivel popular)". En Carbonero Cano, P. (dir.) y M. T. Palet (ed.): Habla de Sevilla y hablas americanas.

Carrasco, Ángeles. 1999. "El tiempo verbal y la sintaxis oracional. La consecutio temporum". En Bosque, Ignacio y Violeta Demonte (eds.): Gramática descriptiva de la lengua española. Vol. 2. Madrid: Espasa-Calpe, 3061-3128.

Crystal, David. 2001. El lenguaje e Internet. Cambridge: Cambridge University Press.

DeMello, George. 1993. “-Ra vs. -Se Subjunctive: A New Look at an Old Topic”. Hispania 1993, 235-244.

DeMello, George. 1997. "Formas verbales en -ra / -se con valor de condicional". En De Kocke, Josse \& George DeMello: Lengua escrita y habla culta en América y España. Diez casos. Salamanca: Ediciones Universidad Salamanca, 39-51.

Grevisse, Maurice. 1993. Le bon usage. Grammaire française. Refondue par André Goosse (13e édition revue). Paris: Duculot.

Kempas, Ilpo. 2011. "Sobre la variación en el marco de la libre elección entre cantara y cantase en el español peninsular". Moenia 17, 243-264.

. 2013. "El uso y la omisión del artículo determinado en grupos relativos preposicionales en español: las propiedades léxico-semánticas del antecedente y la estructura infinitiva del grupo relativo como fuentes de variación". En E. Havu \& I. Hyvärinen (eds.): Comparing and contrasting syntactic structures. From dependency to quasi-subordination. Mémoires de la Société Néophilologique, 86. Helsinki : Société Néophilologique, 1-16. . 2015. "Kielentutkijat ja -käyttäjät keskellä paradigman muutosta: hakukonepohjaisen internet-tiedonhaun mahdollisuudet ja haasteet". En Ari Haasio, Minna Zechner \& Seliina Päällysaho (eds.): Internet, verkkopalvelut ja tietotekniset ratkaisut opetuksessa ja tutkimuksessa. Publications of Seinäjoki University of Applied Sciences. A. Research reports 22. 203-213.

Kempas, Ilpo y López Samaniego, Anna. 2011. "Sobre la elección entre canté y he cantado en presencia de hace x horas en el español peninsular”. Onomázein, 24 (2011/2). 125153. Disponible en www.onomazein.net/24.html [Consulta 18.7.2015].

Labov, William. 1966. The Social Stratification of English in New York City. (Sec. ed. 2006). Washington DC: Center for Applied Linguistics.

López Morales, Humberto. 2004. Sociolingüistica. Tercera edición ampliada. Madrid: Gredos. 
López Samaniego, Anna. 2015. "Del umbral de la acción al umbral del razonamiento. El valor epistémico del operador de entrada". En Borreguero, Margarita y Gómez-Jordana, Sonia (eds.): Les marqueurs du discours dans les langues romanes: une approche contrastive, Limoges: Lambert-Lucas, 137-154.

Lorenzo, Emilio. 1980. El español de hoy, lengua en ebullición. Madrid: Gredos. Mondéjar, José. 1970. El verbo andaluz. Formas y estructuras. Madrid: C.S.I.C. Pottier, Bernard. 1969. Grammaire de l'espagnol. Paris: Presses Universitaires de France.

Quedada Pacheco, Miguel Ángel. 2002. El español de América. Cartago: Editorial Tecnológica de CR.

Real Academia Española. Corpus de referencia del español actual (CREA). Disponible en http://corpus.rae.es/creanet.html [Consulta 4/1/2016].

Real Academia Española y Asociación de Academias de la Lengua Española. 2010. Nueva gramática de la lengua española (NGLE). (Segunda tirada, corregida). Madrid: Espasa-Calpe.

Ridruejo, Emilio. 1999. Modo y modalidad. El modo en las subordinadas sustantivas. En Bosque, Ignacio y Violeta Demonte (eds.): Gramática descriptiva de la lengua española. Vol. 2. Madrid: Espasa-Calpe, 3209-3251.

Rojo, Guillermo. 1978. "La temporalidad verbal en español”, Verba, 1, 68-149. . 1996. "Sobre la distribución de las formas llegara y llegase en español actual". En Casado Velarde, M. et al. (eds.): Scripta Philologica in memoriam Manuel Taboada Cid. A Coruña: Universidade da Coruña, 677-691.

. 2011. "Me pidieron que \{reseñara $\sim$ reseñase\} el libro que Bosque \{?publicara / *publicase\} en 1980". En Escandell, Ma V. et al. (eds.): 60 problemas de gramática dedicados a Ignacio Bosque. Madrid: Akal, 213-219.

Rojo, Guillermo y Veiga, Alexandre 1999. “El tiempo verbal. Los tiempos simples”. En Bosque, Ignacio y Violeta Demonte (eds.): Gramática descriptiva de la lengua española. Vol. 2. Madrid: Espasa-Calpe, 2867-2934.

Sarmiento, Ramón y Sánchez, Aquilino. 1989. Gramática básica del español: norma y uso. Madrid: SGEL-Educación.

Schmidely, Jack. 1992. "Los subjuntivos -ra y -se en oraciones condicionales en Cinco horas con Mario". En Vilanova A. (ed.): Actas del X congreso de la Asociación Internacional de Hispanistas. Vol. 4. Barcelona: Promociones y publicaciones universitarias, 13011311. Disponible en http://cvc.cervantes.es/literatura/aih/pdf/10/aih_10_4_052. pdf [Consulta el 3/1/2016].

Serrano, María José. 1994. La variación sintáctica: formas verbales del periodo hipotético en español. Madrid: Entinema.

. 1996. "El subjuntivo -ra y -se en oraciones condicionales". Estudios filológicos 31, $129-140$.

. 2006. Gramática del discurso. Madrid: Ediciones Akal.

Sinner, Carl. 2008. "La conjugación de los verbos irregulares, entre prejuicio y análisis lingüístico”. En C. Sinner \& A. Wesch (eds.) El castellano en las tierras de habla catalana. 
Madrid - Frankfurt am Main: Iberoamericana - Vervuert, 233-268.

Urrutia Cárdenas, Salvador H. 1988. Esquema de morfosintaxis histórica del español. Bilbao: Publicaciones de la Universidad de Deusto.

Valeš, Miroslav. 2006. "El prestigio desigual de las formas del imperfecto de subjuntivo cantaral cantase". En Moya Corral, J. A. y M. Sosinksi (eds.): Lexicografia y enseñanza de la lengua española. Actas de las XI Jornadas sobre la enseñanza de la lengua española. (Granada). Salamanca: Ediciones de Universidad de Salamanca, 303-3011. Disponible en http://www.ugr.es/-hum430/Actas_XI_Jornadas.pdf [Consulta el 28/12/2015].

Veiga, Alexandre. 1996. La forma verbal española cantara en su diacronía. Universidade de Santiago de Compostela.

Veiga, Alexandre y Mosteiro Louzao, Manuel. 2006. El modo verbal en cláusulas condicionales, causales, consecutivas, concesivas, finales y adverbiales de lugar, tiempo y modo. Salamanca: Ediciones de Universidad de Salamanca.

Yus, Francisco. 2010. Ciberpragmática 2.0. Barcelona: Ariel. 